\title{
Detection of Directed Connectivities in Dynamic Systems for Different Excitation Signals using Spectral Granger Causality
}

\author{
Christian Kühnert ${ }^{1}$, Christian Frey ${ }^{1}$ and Ruben Seyboldt ${ }^{1}$ \\ Fraunhofer Institute of Optronics, System Technologies and Image Exploitation IOSB \\ Fraunhoferstraße 1, 76131 Karlsruhe, Germany \\ \{christian.kuehnert, christian.frey, ruben.seyboldt\}iosb.fraunhofer.de
}

\begin{abstract}
Industrial plants usually consist of different process units which are strongly cross-linked to each other. This leads to the point that a voluntary or involuntary change in one unit (e.g. changing some process control parameter or having a malfunctioning value) can lead to unexpected results in another process unit. Hence, knowing which are the causing and which are the effecting process variables is of great interest. Still, depending on the underlying process and the characteristics of the excitation signal, directed connectivities can or can not be detected.

Therefore, in this paper several types of dynamic SISO systems and excitation signals are defined for which a directed connectivity from input to output signal should be detected and from output to input should not be detected. As a method for the detection of directed influences Spectral Granger Causality is used, which has been extended with a surrogatebased significance test. This test is used to define if a directed influence exists from one process variable to another.
\end{abstract}

Keywords: Spectral Granger Causality · Detection of Directed Connectivities · Time Series Analysis.

\section{Introduction}

Process control systems at production plants usually consist of a large number of process variables, while the interconnectivity of the variables is not always directly evident. Hence, due to the interconnectivity, if some change, voluntary or on purpose is performed on one unit, this can lead to unwanted effects at another unit. Therefore, it is of great interest to understand which variable has a significant influence on another variable.

For the automatic detection of directed connectivities in time series exists already a wide variety of methods, which are mainly developed for the use in neuroscience (e.g. [3] or [1] for reviews) or for the analysis of econometric data [9]. One of the first methods developed, was done by Granger [8], being called the Granger Causality. This method uses two vector autoregressive functions and, by comparing their residual sum of squares, the method tells if one variable causes the other or not. The original approach, taking place in the time domain, 
was extended by Geweke [7] into the spectral domain, having the advantage to select specific frequencies for analysis. In 2000 Schreiber [14] developed a method called Transfer Entropy, which measures the amount of information transferred from one random process to another. In recent research, Transfer Entropy has been extended by contains several extensions like Partial Transfer Entropy [11] or Symbolic Entropy [13]. Bauer [2] proposes a Nearest-Neighbor approach for cause-effect analysis. In [12] different methods for the detection of significant directed influences were developed and compared on several benchmarks, consisting of simulated dynamic systems data, biosignals and on disturbances from a glass forming process. Kaminski [10] proposes the estimation of directed transfer functions.

This aim of this paper to investigate under which circumstances it is possible to detect directed influences in measurements, depending on the excitation signal as well as the underlying dynamic systems. As specific detection method Spectral Granger Causality [7] is used, which is extended with a surrogate-based significance test. In difference to [12], which already defines benchmark processes for the detection of causal dependencies, the current paper focuses more on the excitation signal characteristics.

The paper is structured as follows: Section 2 introduces how directed connectivities can be detected in time series and how Spectral Granger Causality is applied. Additionally, the surrogate-based calculation of the significance threshold is explained. Section 3 describes the defined input signals and dynamic systems for benchmarking, while section 4 discusses the results. Finally, section 5 gives a summary and some ideas for future research.

\section{Detecting directed connectivities in time series}

\subsection{Bivariate Spectral Granger Causality}

The concept of Granger causality (GC) has been originally introduced in the field of economics by Clive Granger in 1969 [8] who used it to determine the relationships of different econometric models. The basic concept of bivariate GC can be explained by assuming the two time series $u[k] \in \mathbb{R}$ and $y[k] \in \mathbb{R}$ with $k=1, \ldots, K$ samples. In that case, the causal connectivity $u \rightarrow y$ is assumed to exist if past values from $u[k]$ and $y[k]$ result in a higher forecast accuracy for $y[k]$ than using only past values from $y[k]$. Mathematically, this is evaluated by comparing two linear vector autoregressive models, while the first one only contains past values of $y[k]$, called the restricted model, and the other one containing past values of $u[k]$ and $y[k]$, called the unrestricted model.

Furthermore, Granger Causality can be easily extended into the multivariate case, while good explanations can be found e.g. in [16] or [4]. Since the developed benchmarks in section 3 compare always one input against one output, for simplicity, multivariate GC will not be explained in this paper.

GC in the time domain: Checking if $u$ causes $y$ or $y$ causes $u$, is in the time domain is done by comparing two linear vectorautoregressive (VAR) models. 
The two VAR models are defined as

$$
\begin{aligned}
& u[k]=\sum_{j=1}^{n} a_{u u}[j] \cdot u[k-j]+\sum_{j=1}^{n} a_{u y}[j] \cdot y[k-j]+e_{u}[k], \\
& y[k]=\sum_{j=1}^{n} a_{y y}[j] \cdot y[k-j]+\sum_{j=1}^{n} a_{y u}[j] \cdot u[k-j]+e_{y}[k]
\end{aligned}
$$

containing the residual covariance matrix being defined as

$$
\Sigma=\left(\begin{array}{cc}
\Sigma_{u u} & \Sigma_{u y} \\
\Sigma_{y u} & \Sigma_{y y}
\end{array}\right)
$$

In $1,2 n$ is the model order, $a_{u u}, a_{u y}, a_{y u}, a_{y y} \in \mathbb{R}^{n}$ contain the model coefficients and $e_{u}[k], e_{y}[k] \in \mathbb{R}$ define the residuals. Finally GC checks the coefficients in $a_{y u}$ (respectively $a_{u y}$ ). If these are significantly different from zero, it is assumed that $u$ causes $y$ (respectively $y$ causes $u$ ). Usually, this is done by comparing the squared-sum of residuals of $e_{u}$ (respectively $e_{y}$ ) with and without taking into account the influencing variable $y$ (respectively $u$ ).

GC in the frequency domain The advantage when working in the frequency domain compared to the time domain is that causal connectivities can be tied to specific frequency bands and one gets better insights in the data. The methodology has been explained in detail in [7] and the main steps are given here for completeness. The Fourier Transformation of the equations 1 and 2 can be written in the following set of equations:

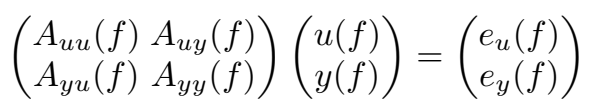

with $u(f)$ and $y(f)$ are the Fourier transformed time series from $u[k]$ and $y[k]$ and $e_{u}(f), e_{y}(f)$ are the Fourier transformations of $e_{u}[k]$ and $e_{y}[k]$. The components of $A$ are then transformed as

$$
\begin{aligned}
& A_{u u}(f)=1-\sum_{i=1}^{n} a_{u u}(n) e^{(-i 2 \pi f n)} \\
& A_{u y}(f)=-\sum_{i=1}^{n} a_{u y}(n) e^{(-i 2 \pi f n)}
\end{aligned}
$$

which counts analogous for $A_{y u}(f)$ and $A_{y y}(f)$. Finally, equation 4 can be rewritten as

$$
\left(\begin{array}{l}
u(f) \\
y(f)
\end{array}\right)=\left(\begin{array}{ll}
H_{u u}(f) & H_{u y}(f) \\
H_{y u}(f) & H_{y y}(f)
\end{array}\right)\left(\begin{array}{l}
e_{u}(f) \\
e_{y}(f)
\end{array}\right)
$$


with $H(f)$ defining the transfer function matrix. Following Geweke [7], under the assumption that the covariance $\Sigma_{u y}=0$, the auto spectrum $S_{u u}(f)$ for the time series $u[k]$ can be derived as

$$
S_{u u}(f)=H_{u u}(f) \Sigma_{u u} H_{u u}(f)^{*}+H_{u y} \Sigma_{y y} H_{u y}(f)^{*} .
$$

The asterisk in equation 8 defines the transposed and complex conjugated transfer function. According to Seth [15], equation 8 can finally be divided into an intrinsic part, namely $H_{u u}(f) \Sigma_{u u} H_{u u}(f)^{*}$ and a causal part, namely $H_{u y} \Sigma_{y y} H_{u y}(f)^{*}$. Hence, the Granger Causality for each frequency can be calculated as

$$
\mathrm{f}_{u \rightarrow y}(f)=\ln \left(\frac{\left|S_{u u}(f)\right|}{\left|S_{u u}(f)-H_{u y} \Sigma_{y y} H_{u y}(f)^{*}\right|}\right) .
$$

Finally, the causal strength $\mathcal{F}_{u \rightarrow y}$ is calculated by integrating over the complete frequency spectrum being defined as

$$
\mathcal{F}_{u \rightarrow y}=\frac{1}{2 \pi} \int_{0}^{2 \pi} \mathrm{f}_{u \rightarrow y}(f) d f
$$

\subsection{Threshold}

The in equation 9 defined causal strength $\mathcal{F}_{u \rightarrow y}$ is not bounded, meaning that from the bare value it is not possible to tell if a causal dependency is really significant or not. Therefore, a threshold needs to be calculated each time an input $u$ is tested against a possible output $y$. Following Choudhury [5] a surrogate time series needs to calculated for $u$, while surrogate means that the phase coupling is removed but the signal keeps the same power spectrum. In other words, all causal information is removed from the signal. To calculate the surrogate of $u$ the following steps need to be performed

$$
\begin{aligned}
& u_{\mathrm{FFT}}=\operatorname{FFT}(u) \\
& u_{\mathrm{FFT}}^{\text {surr }}= \begin{cases}u_{\mathrm{FFT}}[k] & k=1, N / 2+1 \\
u_{\mathrm{FFT}}[k] e^{j \Phi_{k-1}} & k=2, \ldots, N / 2 \\
u_{\mathrm{FFT}}[k] e^{j \Phi_{k-1}} & k=(N / 2+2), \ldots, N\end{cases} \\
& u^{\text {surr }}=\operatorname{IFFT}\left(u_{\mathrm{FFT}}^{\mathrm{surr}}\right)
\end{aligned}
$$

with FFT being the Fourier and IFFT being the Inverse Fourier Transform. In that case $N$ describes the number of samples and $\Phi_{n} \in 0, \ldots, 2 \pi$ with $k=$ $1, \ldots,(N / 2-1)$ is a random phase value. The final threshold is derived in terms of a $3 \sigma$ test being defined as

$$
\mathcal{F}_{u \rightarrow y}^{\text {Threshold }}=\mu^{\text {surr }}+3 \sigma^{\text {surr }}
$$

with

$$
\mu^{\text {surr }}=\frac{1}{M} \sum_{k=1}^{M} \mathcal{F}_{u^{\text {surr }} \rightarrow y}, \quad \sigma^{\text {surr }}=\sqrt{\frac{1}{M} \sum_{m=1}^{M}\left(\mathcal{F}_{u^{\text {surr }} \rightarrow y}-\mu^{\text {surr }}\right)^{2} .}
$$


and $M$ being the number of surrogate trials. If the outcome indicates $\mathcal{F}_{u \rightarrow y}>$ $\mathcal{F}_{u \rightarrow y}^{\text {Threshold }}$, the found causal dependency is defined as being significant.

\section{Benchmarks}

For the detection of directed connectivities in time series two things are important, namely the characteristics of the excitation signal and the underlying process behavior. Hence, this section proposes several possible input signals (section 3.1) and several dynamic SISO systems (section 3.2). Next, the Spectral Granger Causality is used to detect the input and output signal for each pair.

\subsection{Analyzed Excitation Signals}

As excitation signals white noise, a sinusoid, the sawtooth wave, an impulse train and a time series based on a random walk are used. All signals are shown in figure 1 in the time domain as well as its power spectrum. For analysis, each signal consists in summary of $N=1000$ samples. The details of the excitation signals are as follows:

White Noise - A time series that consists of white noise means to have a sequence of uncorrelated random variables with constant mean $\mu$ and variance $\sigma^{2}$. In the following, the input time series $u_{\mathrm{wn}}[k] \in \mathbb{R}$ is modeled as a stochastic process with $\mu=0$ and $\sigma^{2}=1$.

Sinusoid - A sinusoid can be seen as a prototype of a periodic disturbance, resulting e.g. from poorly tuned PI-controllers. For the input series a sinusoid of the form $u_{\sin }[k]=\sin (\omega \cdot k) \in \mathbb{R}$ is used with an angular frequency of $\omega=2 \pi \cdot 0.1$.

Sawtooth Wave - This time series can be interpreted as some sort of a drift e.g. when sensors are slowly polluting. For the sawtooth wave the input series $u_{\mathrm{sw}}[k] \in \mathbb{R}$ is defined as $u_{\mathrm{sw}}[k]=\operatorname{frac}\left(\frac{k}{T}+\Phi\right)$ with a period of $T=100$ and the phase $\Phi=0$ and frac being the fractional part defined as frac $\equiv x-\lfloor x\rfloor$.

Impulse train - Having so-called impulse or spike train means that e.g. an inert gas or fluid injection into a process at a predefined cycle occurs. Therefore, the input time series $u_{\text {it }}[k] \in \mathbb{R}$ is defined as $u_{\text {it }}[k]=\sum_{k=0}^{\frac{N}{K}-1} \delta[n-k K]$ with $N \mid K$, $\delta$ being a Dirac impulse, $N \in \mathbb{R}$ representing the length of the time series and $K \in \mathbb{R}$ the period. In the following the period $K$ is set to 100 .

Random Walk - The time series of a random walk is defined as a process where the value at sample point $[k]$ is composed of the past value $[k-1]$ plus an error term defined as white noise. In this paper the random walk is used to investigate how used methods behave on low-frequent changes in a process e.g. when having a fluctuation of some concentration in a fluid. Therefore, the input time series $u_{\mathrm{RW}}[k] \in \mathbb{R}$ is defined as $u_{\mathrm{RW}}[k]=u_{\mathrm{RW}}[k-1]+\epsilon[k]$ where $\epsilon[k]$ is a white-noise sequence with $\mu=0$ and $\sigma^{2}=0.1$. 
Time Series
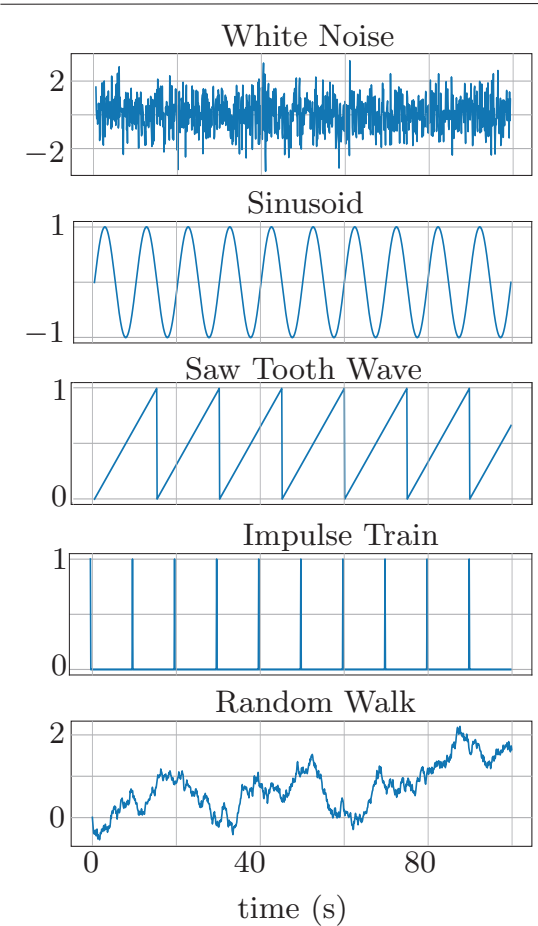

Power Spectrum $(\mathrm{dB})$

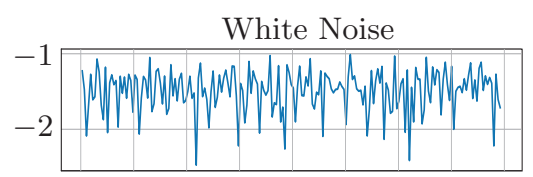

Sinusoid

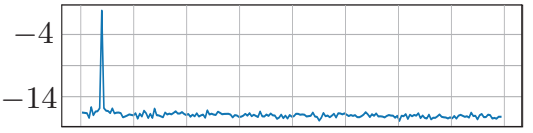

Saw Tooth Wave

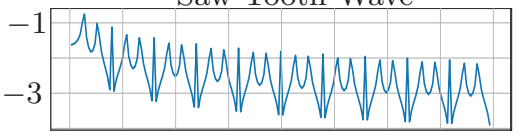

Impulse Train

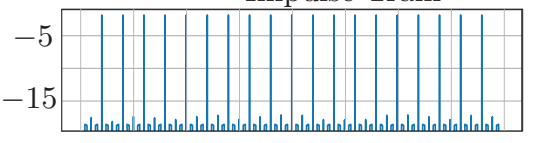

Random Walk

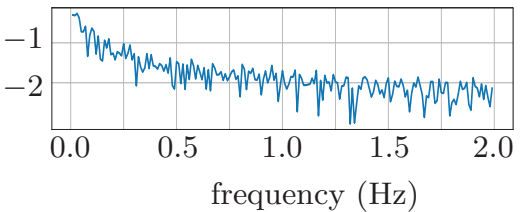

Fig. 1. Investigated excitation signals in the time domain and their corresponding power spectra.

\subsection{Dynamic systems}

Figure 2 shows the selected dynamic systems which are tested in combination with the prior shown excitation signals. In detail, the systems consist of a dead time, a low-pass filter, a nonlinearity and finally a resonant second order system. In detail, the systems are described as follows:

Dead Time - In this benchmark, the excitation signal is shifted by 10 samples. No dynamic system is added between input and output signal. Hence, this responds to the most simple case for the detection of directed connectivities from one signal to another.

Low-pass filter - The low pass filter with the time constant $T=1 \mathrm{~s}$ represents the most basic system for the detection of input and output signal. In process technology low-pass filter are e.g. fluid tanks or pipes which tend to attenuate a disturbance and hence making in sometimes complicated to track back the disturbance propagation path. This benchmark is mainly used to investigate the behavior regarding the defined input signals in section 3.1. 
Nonlinear system - In this process a sinusoid is taken from the intermediate output signal $y_{1}(t)$. Depending on the amplitude of the excitation signal, the sinusoid will have a strong impact on the resulting output signal. The main purpose is to determine for which input signals the methods can still determine the input and output signals and their parameters.

Resonant system - This benchmark represents a classic mass-spring-damper system. Like for the other systems the time constant is set to $T=1 \mathrm{~s}$, while the dimensionless damping ratio is set to $\xi=0.05$.

Dead time

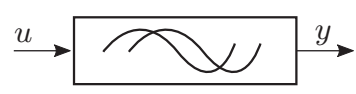

Low-pass filter

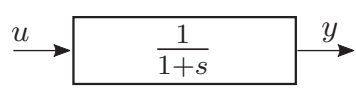

Nonlinear system

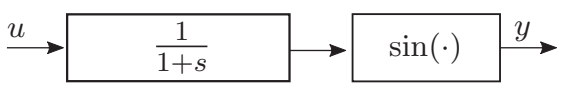

Resonance

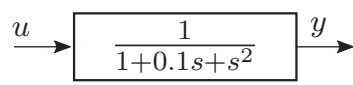

Fig. 2. Used transfer functions for the validation of the detection of directed influences.

\section{Results}

For analysis, each dynamic systems wax excited with the different input signals and the spectral Granger causality was used for the detection of directed influences from $u \rightarrow y$, with results shown in figure 4 , and from $y \rightarrow u$, where the results are shown in figure 4 . If a directed influence has been found, the corresponding box contains a checkmark, elsewise it contains a cross. In the following a summary is given by following corresponding to the defined benchmarking dynamic systems.

Dead time: In that use case, consisting of a simple time shift, for all input signals, the directed dependencies from $u \rightarrow y$ are detected and defined as being significant. Nevertheless, for the input signal $u_{\text {sin }}$ and $u_{\text {imp }}$ a false positive directed influence has been found pointing from $y \rightarrow u$. The explanation is straight forward, since the impulse train as well as the sinusoid are cyclic excitations signals, hence having only a time shift in the signals, it is not possible to distinguish input from output signal . 
Dead time

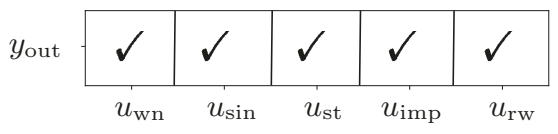

Low-pass filter

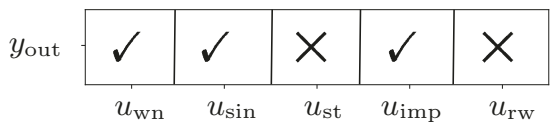

Nonlinear System

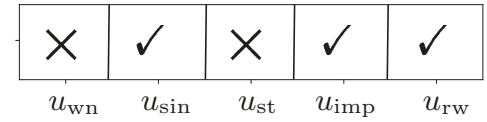

Resonance

$y_{\text {out }}$

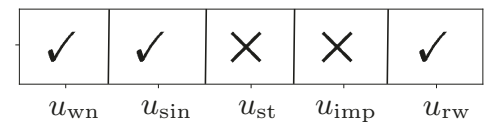

Fig. 3. Results of the benchmarks when testing for directed influences $\mathcal{F}_{u \rightarrow y}$
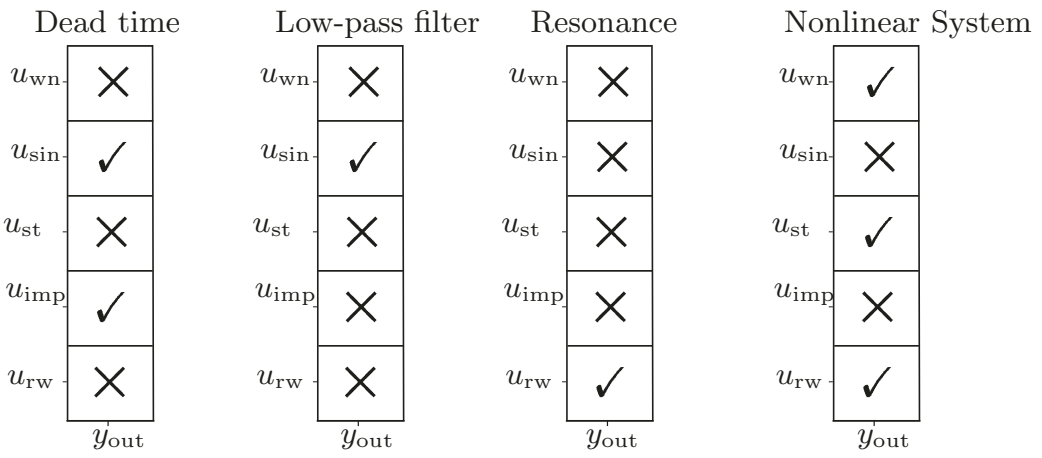

Fig. 4. Results of the benchmarks when testing for directed influences $\mathcal{F}_{y \rightarrow u}$

Low-pass filter: Regarding the low pass filter, $u_{\mathrm{wn}}, u_{\mathrm{sin}}$ and $u_{\mathrm{imp}}$ detect the correct directed connectivity. The saw tooth and random walk, both having a similar power spectrum (see figure 1) are not detected. The reason is that the low-pass filter has to too much attenuation, resulting in an output signal which has already too much information about itself in past values. Hence, in terms of Granger Causality, this results in a non-significant information gain for $u_{\mathrm{rw}}$. The only excitation signal leading to a connectivity from $y \rightarrow u$ is the sinusoid. Like for the dead time benchmark, the reason is that the sinusoid is cyclic.

Nonlinear system: Adding an additional sinusoid as a non-linearity to the lowpass filter in the prior benchmark changes the results of the detected directed influences significantly. $u_{\mathrm{wn}}$ does no longer detect the connectivity $u \rightarrow y$, while the $u_{\mathrm{rw}}$ is detecting it. The two excitation signals $u_{\mathrm{sin}}$ and $u_{\mathrm{imp}}$ behave like without non-linearity. Regarding the directed, causal wrong influence $y \rightarrow u$ the excitation signals $u_{\mathrm{wn}}, u_{\mathrm{st}}$ and $u_{\mathrm{rw}}$ detect this influence. Only the $u_{\mathrm{sin}}$ and $u_{\mathrm{imp}}$ correctly define the influence as not significant. 
Resonance: Detecting $u \rightarrow y$ in the spring-mass-damper benchmark is only possible with $u_{\mathrm{wn}}, u_{\mathrm{sin}}$ and $u_{\mathrm{rw}}$. When having as excitation the signals $u_{\mathrm{imp}}$ and $u_{\text {st }}$, spectral Granger Causality assumes that there is neither a directed influence from $u \rightarrow y$ nor from $y \rightarrow u$. Furthermore, except for $u_{\text {rw }}$ none of the excitation signals detect in a wrong causal influence $y \rightarrow u$.

\section{Summary}

The results showed when using spectral Granger Causality, the detection of directed influences in time series depends the excitating signal as well as on the underlying dynamic system. Regarding the excitation signals, for none of the signals it was possible to detect for all four dynamic systems the correct directed influence $u \rightarrow y$, while at the same time never detecting a wrong influence $y \rightarrow u$. Hence, when using Granger Causality, detected or not detected directed influences in data always need to be questioned in terms of the excitation as well as in terms of the underlying process behavior. Still, this method can be of great help to generate first a understanding of the influences variables have onto each other in a data set, since no always, but most of the times Granger Causality detected the correct dependency.

In terms of the development of benchmarks, there is a variety of future research. Questions that arise are the impact of noise in the data or how a directed influence can still be detected if variables having a common cause. Regarding Granger Causality, it can be evaluated, in which cases it is possible to differenciate between direct and indirect influences, e.g. when using the multivariate Granger Causality. Additionally, the benchmarks should be used to compare several methods like Transfer Entropy with its extensions or the estimation of Directed Transfer Functions.

\section{Acknowledgements}

This work was developed in the Fraunhofer Cluster of Excellence "Cognitive Internet Technologies".

\section{References}

1. Bastos, A. and Schoffelen, J.: A Tutorial Review of Functional Connectivity Analysis Methods and Their Interpretational Pitfalls, Frontiers in Systems Neuroscience(9), pp. 175, (2016)

2. Bauer, M.: Data-driven Methods for Process Analysis. University College London, PhD Thesis, (2005)

3. Blinowska, K.: Review of the methods of determination of directed connectivity from multichannel data, Medical \& Biological Engineering \& Computing (49), pp. $521-529,(2011)$

4. Blinowska, K. et al.: Granger causality and information flow in multivariate processes, Phy. Rev. E.(70), no.p. 4, (2004) 
5. Choudhury, A.A.S. and Shah, S.L. and Thornhill, N.F.: Diagnosis of Process Nonlinearities and Valve Stiction: Data Driven Approaches, Advances in Industrial Control, Springer, (2008)

6. Spectral connectivity toolbox: "https://github.com/Eden-Kramer-Lab/spectral_ connectivity/", last access August 2018

7. Geweke, J., Measurement of linear dependence and feedback between multiple time series. Journal of American Statistical Association, vol. 77. pp 304-313 (1982)

8. Granger, C. W.J.: Investigating Causal relations by Econometric Models and CrossSpectral Methods, Econometrica 37, (1969)

9. Heckman, J.: Econometric causality, International statistical review(76), pp. 1-27, (2008)

10. Kaminski M., and Blinowska K.J.: Directed Transfer Function is not influenced by volume conduction - inexpedient pre-processing should be avoided, Frontiers in Computational Neuroscience. (8), (2014)

11. Kugiumtzis, D.: Partial transfer entropy on rank vectors, The European Physical Journal Special Topics (222), pp. 401-420, (2013)

12. Kühnert, C., et al.: Methoden zur datengetriebenen Formulierung und Visualisierung von Kausalitätshypothesen. at - Automatisierungstechnik Methoden und Anwendungen der Steuerungs-, Regelungs- und Informationstechnik, 60(10), pp. 630-640. , (2012)

13. Staniek, M. and Lehnertz, K.: Symbolic transfer entropy: inferring directionality in biosignals,Biomedizinische Technik/Biomedical Engineering (54), pp.323-328, (2009)

14. Schreiber, T.: Measuring information transfer, Physical Review letters 85(2), pp. 461-464, (2000)

15. Seth, A. et al.: Granger Causality Analysis in Neuroscience and Neuroimaging, Journal of Neuroscienc(8), pp. 3293-3297, (2015)

16. Yang, D. et al.: Granger Causality for Multivariate Time Series Classification, IEEE International Conference on Big Knowledge (ICBK), pp. 103-110, (2017)

Open Access This chapter is licensed under the terms of the Creative Commons Attribution 4.0 International License (http://creativecommons.org/licenses/by/4.0/), which permits use, sharing, adaptation, distribution and reproduction in any medium or format, as long as you give appropriate credit to the original author(s) and the source, provide a link to the Creative Commons licence and indicate if changes were made

The images or other third party material in this chapter are included in the chapter's Creative Commons licence, unless indicated otherwise in a credit line to the material. If material is not included in the chapter's Creative Commons licence and your intended use is not permitted by statutory regulation or exceeds the permitted use, you will need to obtain permission directly from the copyright holder.

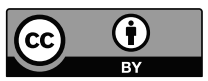

